\title{
FONDATEURS ET COLLABORATEURS, LES DÉBUTS DE LA REVUE DE SYNTHÈSE HISTORIQUE (1900-1910)
}

\author{
Martin FugLER
}

Lorsque au mois d'août 1900 paraît le numéro un de la Revue de synthèse historique, Henri Berr a terminé sa thèse depuis deux ans, une thèse qu'il a intitulée: L'Avenir de la philosophie, esquisse d'une synthèse des connaissances fondée sur l'histoire ${ }^{1}$. Cinquante ans après, dans son dernier livre, et peu avant sa mort, celui-ci écrira : «Ce titre exprimait ce qui était déjà ma double préoccupation, ce à quoi ont répondu dans le demisiècle suivant toutes les ouvres que j'ai pu créer ${ }^{2}$.»

La Revue de synthèse historique fut donc la première de ses « auvres », la première mise en pratique de ses idées. Henri Berr présenta ainsi son périodique: «Quel que soit le nombre des revues existantes, celle qu'on annonce aujourd'hui au public ne fera double emploi avec aucune. Elle est neuve par son objet et par son caractère. Elle répond à un besoin du temps. Elle est appelée à rendre des services divers ${ }^{3}$. »

À qui la revue pourrait-elle rendre ces « services divers » ? G. Monod, alors directeur de la Revue historique, ne se sentait pas concerné et déclara dans un entretien au Temps : «Quelques philosophes [...] ont une tendance à se mêler d'histoire, à faire de la généralisation historique. Monsieur Berr va fonder une Revue de Synthèse Historique [...]. Cette revue rencontrera peut-être plus d'adhérents chez les philosophes que chez les historiens ${ }^{4}$. » L'historiographie a

1. Publiée en $1899 \mathrm{chez}$ Hachette (sa thèse latine a pour titre : An jure inter scepticos Gassendus numeratus fuerit).

2. Henri Berr, La Montée de l'esprit, Paris, A. Michel, 1955, p. 138.

3. Second prospectus de présentation, publié (au moins partiellement) dans la Revue de synthèse à l'occasion de son cinquantenaire. Revue de synthèse, t. 67, 1950, p. 5. La Revue de synthèse historique (abrégée RSH) est devenue la Revue de synthèse en 1930; dans ces notes c'est la numérotation de la « série générale » qui a été retenue (t. 1 en 1900).

4. Gabriel MONOD, dans un entretien avec E. Fazy, Le Temps, 5 sept. 1900. 

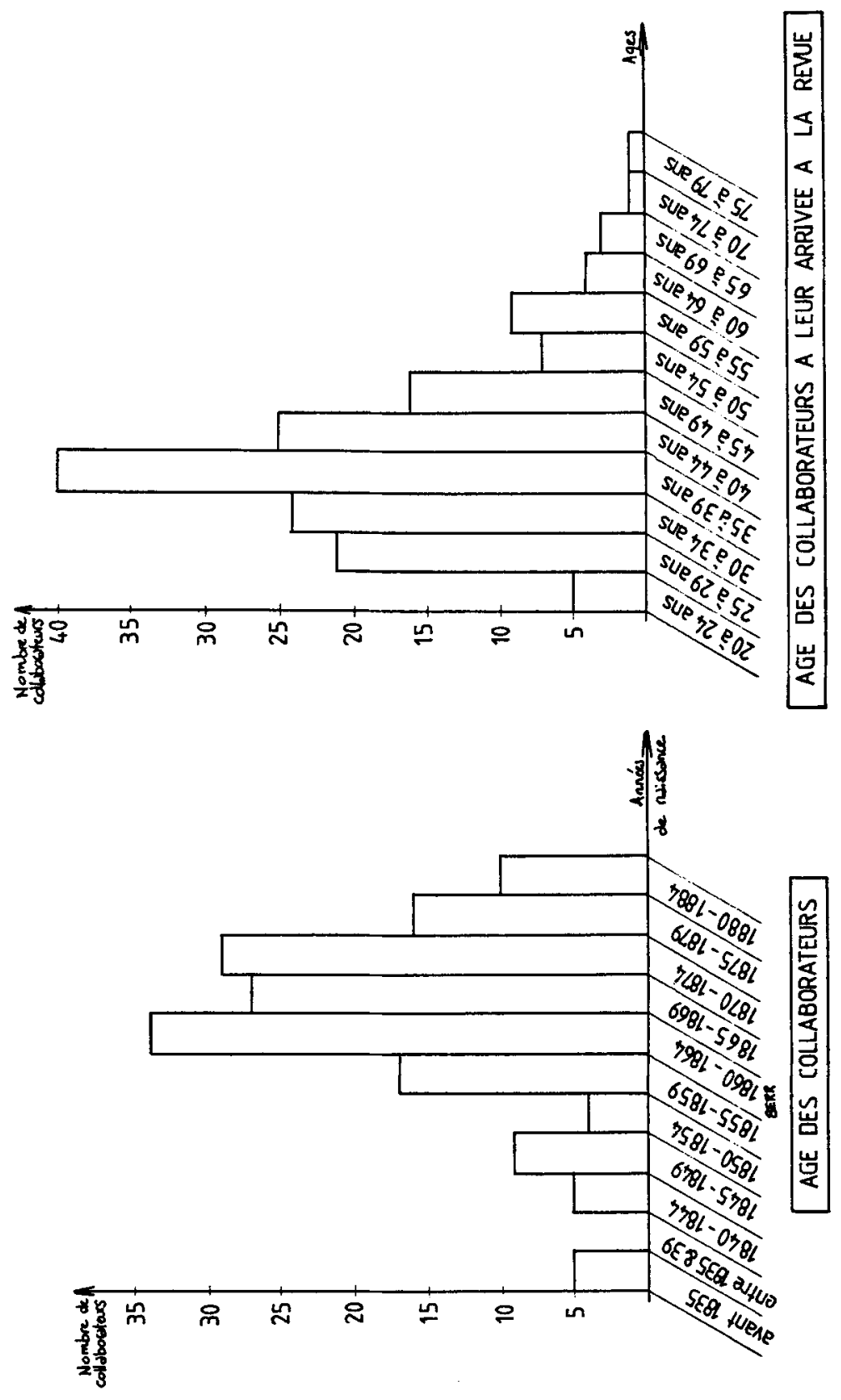
entériné cet a priori: M. Siegel, par exemple, affirme que «le jugement de Monod au sujet du projet d'Henri Berr s'est révélé incroyablement juste » et il parle des « quelques historiens qui s'intéressèrent aux idées d'Henri Berr ${ }^{5}$ ».

À qui s'adressa cette revue de «Synthèse historique » et qui Henri Berr sut-il intéresser à son entreprise ? Pour y répondre, trois angles de vue successifs seront adoptés : dans un premier temps la revue telle qu'Henri Berr l'a conçue et dirigée, puis telle qu'elle a été réalisée par ses collaborateurs et finalement, telle que les autres l'ont perçue afin de mettre en lumière l'influence d'Henri Berr sur la pratique historique de ses contemporains comme de leurs successeurs.

Différentes périodisations étaient susceptibles d'être retenues pour délimiter l'étude de la revue : son interruption due à la Première Guerre mondiale, son changement de nom en 1930 , etc. Celle qui a paru la plus adéquate va de son lancement à 1910 , date à laquelle Henri Berr dressa le premier bilan de son bimestriel, suivi, en 1911, par un livre présenté comme le fruit des dix années de travail de sa revue et reconnu comme son ouvrage le plus important : La Synthèse en histoire. De plus, cette décennie, malgré les vingt volumes publiés, constitue un ensemble suffisamment restreint pour permettre une analyse approfondie ${ }^{6}$.

La Revue de synthèse historique avait un programme précis, longuement présenté — avant même sa parution ${ }^{7}$ — aux lecteurs comme aux collaborateurs éventuels. Il visait quatre objectifs : rapprocher les chercheurs des différentes disciplines, les faire collaborer, pour constater l'état de la recherche (en rejetant le non-scientifique - le vague, l'arbitraire) afin de synthétiser ces connaissances dispersées ${ }^{8}$. En effet, pour Henri Berr, «il [était] temps de réagir [...] contre les excès de l'analyse où se perd la notion de but à atteindre ${ }^{9} »$. Paul Lacombe, qui devint rapidement l'un des principaux collaborateurs de la revue, résume ainsi la situation des études historiques au tournant du siècle :

5. Martin SIEgEL, «Henri Berr et la Revue de synthèse historique », in Au Berceau des Annales, Ch.-O. Carbonell et G. Livet (éd.), Toulouse, Presses de l'IEP, 1983, p. 205-218.

6. L'ensemble des éléments publiés ici sont issus de mon mémoire de maîtrise réalisé a Strasbourg en 1985 sous la direction de François HARTOG et Pierre AYÇOBERRY, Analyse ou synthèse? La Revue de Synthèse historique et l'histoire (1900-1910). Il a été déposé au Centre international de synthèse.

7. Art. cit. supra n. 3. Le premier prospectus a également été publié ultérieurement à l'occasion du centenaire de la naissance d'Henri Berr (Revue de synthèse, t. 85, 1964, p. 104). Ce programme est commenté en tête du premier numéro de la revue (H. BERR, «Sur notre programme ", $R S H$, t. 1,1900 , p. 1-8).

8. Art. cit. supra n. 3, p. 6 .

9. H. BERR, lettre à Gaston Paris, Bibliothèque nationale, NAF, 24432, fol. 33-35. 
«Tenter la constitution de l'histoire science est un ouvrage qui s'impose à notre temps, il s'agit non seulement d'utiliser des matériaux en nombre immense, dont jusqu'ici le profit est presque nul; mais il y a surtout urgence à alléger l'esprit humain d'un faix qui devient écrasant. On ne diminue le poids de phénomènes qu'en les liant, et ce lien ne peut être qu'une généralisation scientifique ${ }^{10}$.»

Pour réaliser ce programme, la revue fut, au départ, scindée en quatre rubriques: les Articles (sur la théorie et la méthode); les Revues générales («inventaires du travail fait et à faire »); la Bibliographie (analyse critique, sous diverses formes, de nouvelles publications) et les Notes, questions et discussions (qui devaient contribuer à développer les relations dans le domaine intellectuel par l'échange d'informations et de points de vue). Elles ne tardèrent pas à se développer, à se subdiviser. C'est ainsi qu'en 1902 (dans le tome IV) fut lancée la première Enquête et en 1903 (dans le tome VI) débutèrent des «études de la psychologie provinciale » : les Régions de France. Deux types de recherches qui devaient favoriser la collaboration en organisant les travaux individuels puisque procédés et domaines d'investigation étaient prédéterminés : susciter des monographies était pour Henri Berr la meilleure façon d'éviter la dispersion des efforts puisque cela garantissait un bon ajustement des différents travaux ${ }^{11}$.

Les études bibliographiques faisaient le point sur ce qui était réalisé dans le domaine historique; les informations transmises, les discussions devaient permettre de suivre ce qui était en train de se réaliser; les essais de synthèse - quels qu'ils soient - avaient pour objectif de faire avancer les recherches. À côté des bilans, des essais synthétiques, il s'avérait nécessaire de mettre à profit les résultats acquis et de constater ce qui restait à faire, dresser un « inventaire du travail fait et à faire » fut le rôle des Revues générales. Prévues pour parcourir «successivement TOUT le domaine de l'érudition, l'histoire entière de la pensée et de l'action humaine ${ }^{12}{ }^{2}$, elles devaient permettre à «tous les esprits curieux d'embrasser dans une seule publication tout l'horizon historique ", aux historiens spécialistes d'être informés des résultats des spécialités voisines et aux « travailleurs novices ou isolés de ne pas gaspiller leurs efforts » mais « de choisir à bon escient leur tâche et de faire une besogne utile ${ }^{13}$ »: «Si l'on souhaite que l'esprit

10. Paul Lacombe, De l'histoire considérée comme science, Paris, Hachette, 1894, p. XI.

11. «Il ne suffit pas de répertorier le travail accompli, il convient de régler le travail qui s'accomplit. » (H. BERR, «Introduction générale des Régions de France », RSH, t. 6, 1903, p. 167.

12. Premier prospectus, art. cit. supra n. 7. Les majuscules sont dans le texte.

13. Second prospectus, art. cit. supra n. 3. 


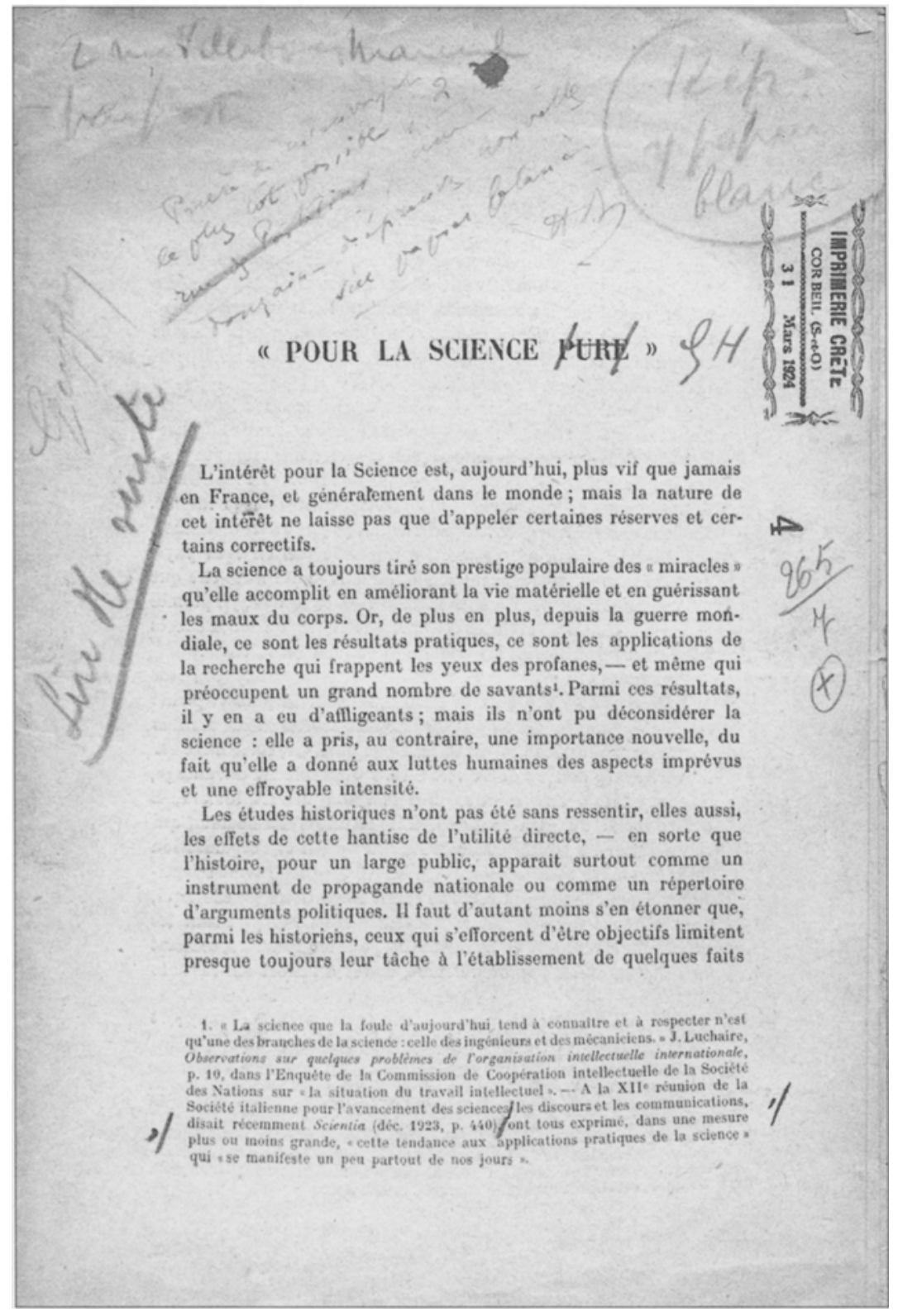

Epreuve de l'article d'Henri Berr annonçant la création du Centre de synthèse, 1925. 


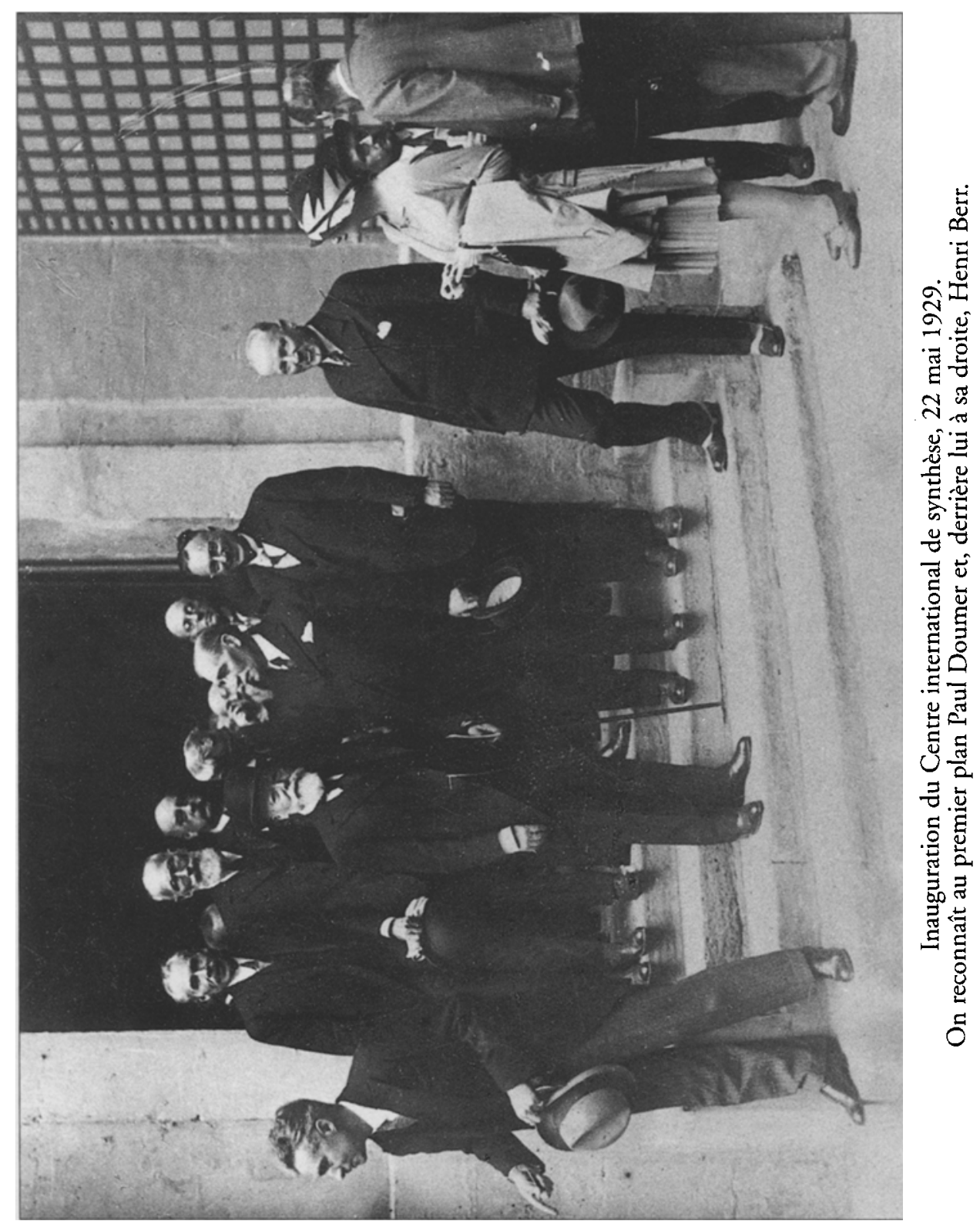




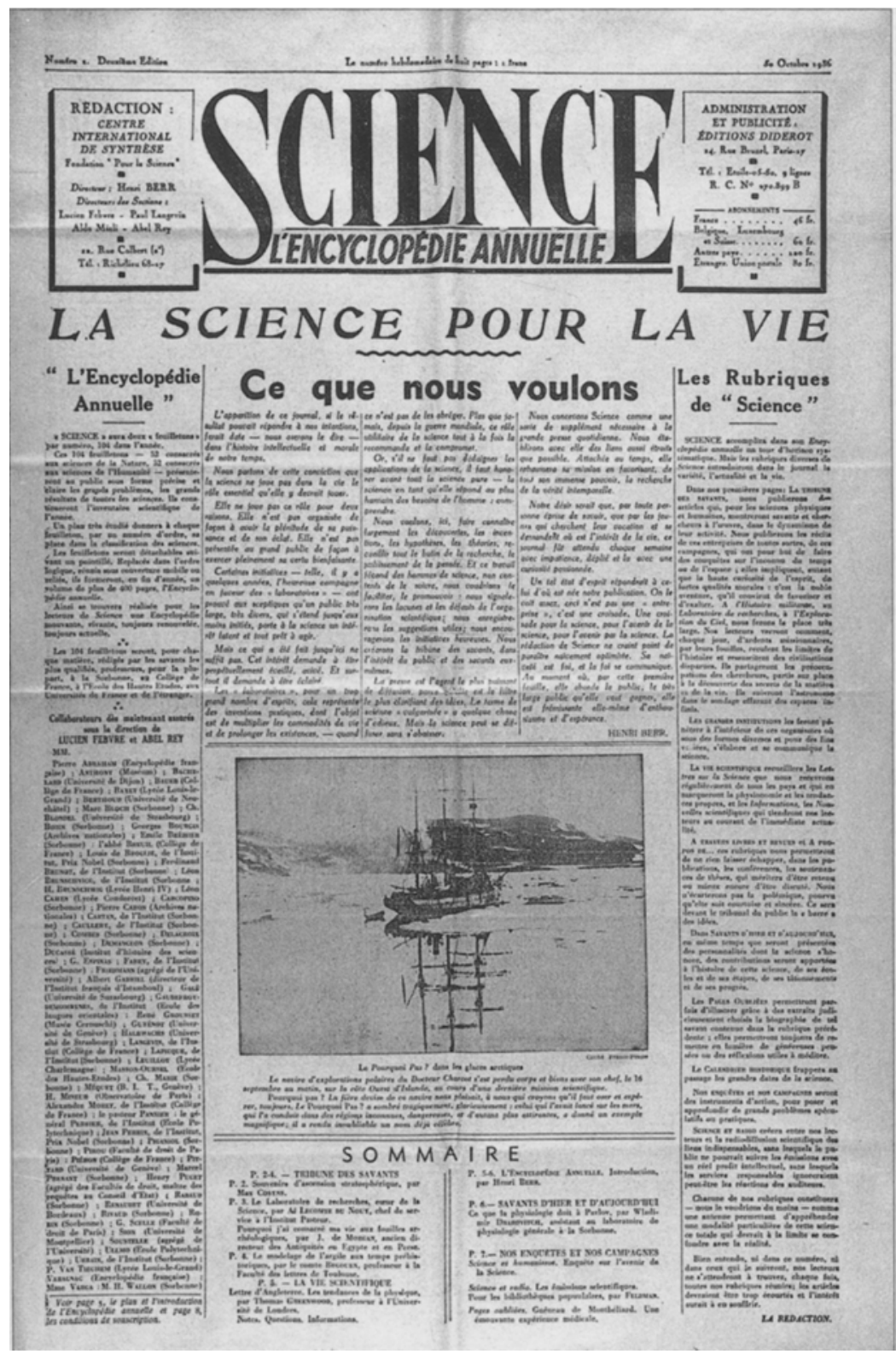

Premier numéro du journal Science, 15 octobre 1936. 


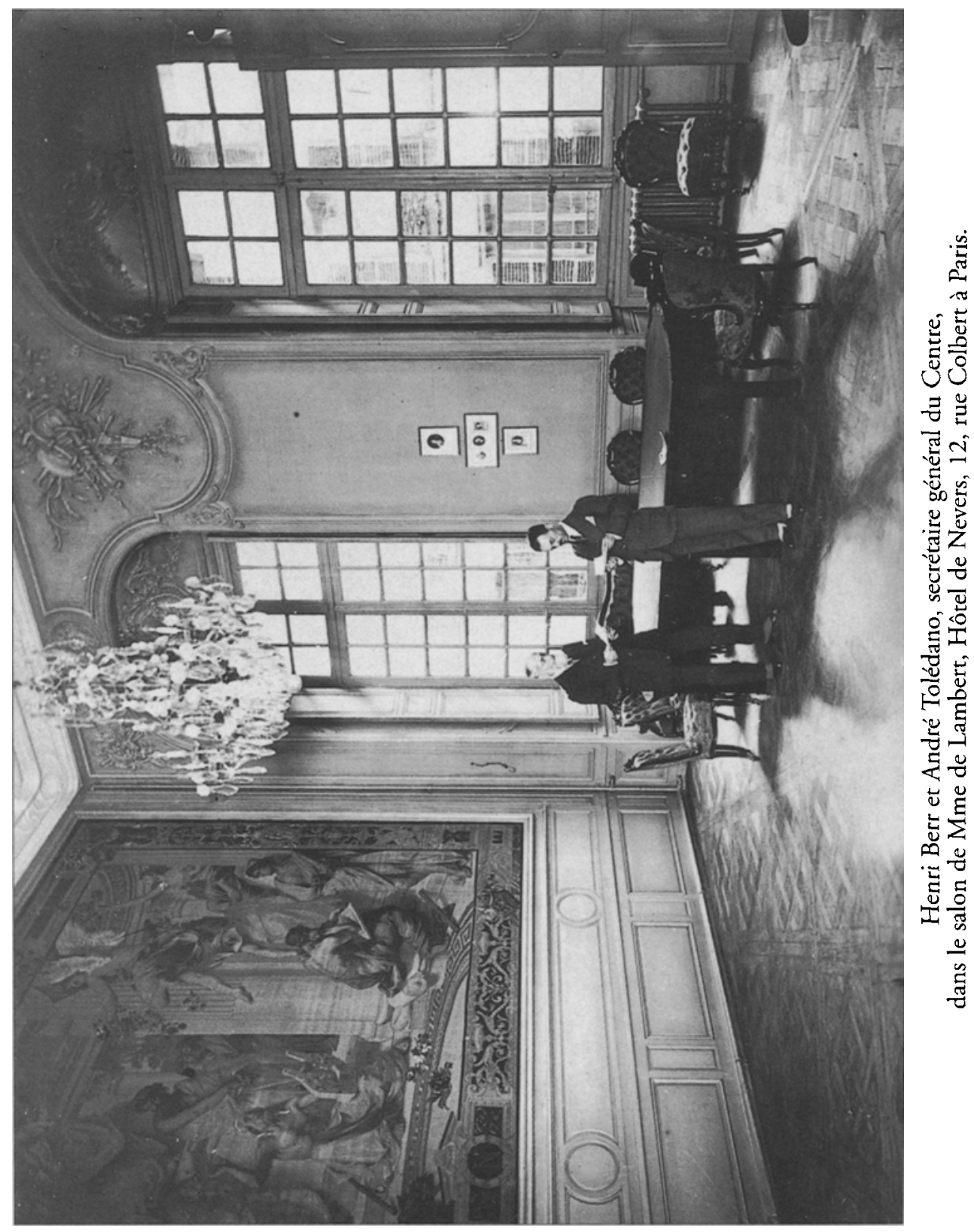


de synthèse descende de plus en plus dans l'analyse pour la rendre plus efficace, plus consciente, plus joyeuse, on veut que la précision, que la rigueur des travaux analytiques, subsiste dans les essais de synthèse ${ }^{14}$ », précisait le directeur de la Revue de synthèse historique. En s'organisant autour d'un triple questionnement ( Quels sont les résultats acquis, quelles sont les questions controversées et quelles sont les lacunes à combler ${ }^{15}$ ?»), ces Revues générales devaient signaler «au lieu de les dissimuler», les "lacunes de la connaissance ${ }^{16}$ ", les mettre en évidence afin de permettre d'y remédier, afin de permettre aux autres de les combler : il ne fallait plus protéger son domaine mais le partager!

Pour comprendre comment, concrètement, s'organisait le travail de cette revue, il faut dépasser le cadre strict des signataires. En effet, comme dans toutes les réalisations d'Henri Berr, le rôle de ses cercles de relation (dans les milieux intellectuels mais aussi bancaires, financiers ou politiques ${ }^{17}$ ) a été essentiel et les allusions à une autre forme de collaboration sont fréquentes sous sa plume (à propos de Frédéric Rauh, Henry Michel ou Octave Gréard par exemple) ${ }^{18}$.

Ainsi, les réunions au siège de la revue, au « 12 rue Sainte-Anne », semblent avoir été déterminantes. Elles étaient hebdomadaires et, à en croire les différents témoignages, souvent animées. Elles sont présentées comme des institutions, avec des habitués dont Paul Lacombe, Abel Rey ou Félix Mathieu - qui pourtant ne signa aucun article dans la revue - et des visiteurs, vieux ou jeunes, «puits de science» ou «minces débuitants » (L. Febvre ${ }^{19}$ ). Les idées les plus abstraites comme les points les plus concrets du fonctionnement d'un périodique y étaient abordés. C'était en quelque sorte le club, le cercle des collaborateurs autour duquel s'échafaudait la Revue de synthèse historique.

À côté des discussions formelles, le fonctionnement de la revue devait être minutieusement organisé car Henri Berr parle de «chefs d'équipe éprouvés » dont dépendait la bibliographie et qui étaient libres de choisir,

14. « Sur notre programme », art. cit. supra n. 7, p. 7.

15. Louis REAU, dans une Revue générale d'histoire de l'art, «L'Art allemand», $R S H$, t. 15,1907, p. 50 et 51 . C'est lui qui souligne.

16. H. BERR, La Synthèse en histoire, essai critique et théorique, Paris, F. Alcan, 1911, p. $10-12$.

17. Voir notamment «Plaidoyer pour la culture scientifique » un entretien avec Jacques Roger, alors directeur du Centre international de synthèse, publié dans Le Monde, 23 nov. 1989 , p. 17.

18. H. BeRR, «Frédéric Rauh (1861-1909)», RSH, t. 18, 1909, p. 1-9, et dans deux Notes (RSH, 1904, t. 9, p. 379 et t. 8 , p. 255).

19. Lucien FEBVRE, «Hommage à Henri Berr », Revue de synthèse, t. 85, 1964, p. 8-12. 
pour les aider, «des auxiliaires de leur choix ${ }^{20} »$. Certains collaborateurs étaient très spécialisés et se consacraient exclusivement à un sujet précis qu'ils étaient seuls à traiter : c'est ainsi que E. Goblot se consacra à l'étude critique de L'Année sociologique ou que L. Pineau se réserva toutes les publications qui traitaient de «folklore».

Les portraits d'Henri Berr tracés dans les différents témoignages concordent et présentent le directeur de la Revue de synthèse historique comme un personnage calme, mesuré, direct et étonnamment cultivé; ils signalent son « humanité » et sa bonté. Nombreux sont ceux qui ont été marqués par son bureau - rempli de livres et de papiers - et l'accueil dans sa maison. Ils évoquent une ambiance familiale où Henri Berr incarne le patriarche, barbu, bon père, simple et généreux; dans ce tableau d'intérieur, même Mme Berr, «inspiratrice chérie, le reflet de son mari », tient son rôle ${ }^{21} \ldots$

Cela dit, que la plupart de ces textes soient commémoratifs n'est certainement pas étranger à cette vision idyllique de ce «maître de tous les maîtres qui vont grandir à ses côtés », comme l'a appelé $\mathrm{F}$. Braudel ${ }^{22} \ldots$ Telle devait être, toutefois, l'ambiance générale dans laquelle se mouvaient les proches de la revue. Mais Henri Berr, directeur de périodique, était également - et malgré sa foi en les «vertus souveraines " de la discussion - le maître d'œuvre incontesté de sa revue. Si l'on en croit R. Bouvier, "Berr était diplomate, il possédait l'art [...] de tourner avec adresse un compliment, un remerciement caressant, flatteur sans exagération [...]. Il savait mettre en valeur les connaisseurs, faire taire les bavards, donner toujours aussi sa propre opinion ${ }^{23} »$. Comme le laisse entendre cette définition, Henri Berr eut certainement une main de fer dans un gant de velours, et on peut très certainement induire une généralité de ce qu'il dit en 1947 de l'histoire des sciences: "Quand, il y a près d'un siècle, j'ai créé la Revue de synthèse historique [...] j'ai naturellement fait place à l'histoire des sciences, $j$ 'ai même fait tout de suite à cette histoire une place d'honneur ${ }^{24} » \ldots$ « J'ai .»

Il affirme ailleurs : « Les collaborateurs de la revue savaient qu'ils étaient libres dans l'intérêt de la science et tout ce qui n'était pas de l'intérêt de la science nous était étranger ${ }^{25} »$. Mais qui plaçait les bornes de cette

20. H. BERR, «Programme d'une bibliographie synthétique », RSH, t. 30, 1920, p. 79.

21. Voir Henri Berr (1863-1954), publication du Centre international de synthèse à l'occasion de sa mort (Paris, A. Michel, 1955).

22. Fernand BRAUDEL, «Hommage à Henri Berr », Revue de synthèse, t. 85, 1964, p. 20.

23. Robert Bouvier, Revue de synthèse, t. 85, juil.-sept. 1964, p. 46.

24. H. BERR, «Antécédents à la nouvelle Revue d'histoire des sciences », Revue d'histoire des sciences et de leurs applications, t. 1, 1947, p. 5. C'est moi qui souligne.

25. H. BERR, « Au bout de dix ans», RSH, t. 21, 1910, p. 5. 
« liberté », qui délimitait « l'intérêt de la science » et excluait ce qui n'entrait pas dans cette catégorie si ce n'est Henri Berr? À plusieurs reprises, les publications de la revue laissent transparaître une opposition entre le directeur et un collaborateur qui a du mal à se plier au «cadre de cette revue $^{26} »$. Pour éviter une analyse de texte un peu fastidieuse, c'est un exemple pris hors du cadre strict de cette étude qui illustrera cette image d'Henri Berr maître des entreprises qu'il a lancées : le compte rendu du premier conseil d'administration du Centre de synthèse. Chacune des corrections faites par Henri Berr sur le brouillon de ce compte rendu conforte le portrait tracé par $\mathbf{R}$. Bouvier en prouvant sa diplomatie, son art de tourner les compliments, sa discrète flatterie mais aussi sa capacité à faire taire les bavards : c'est ainsi que les paragraphes relatant les discussions sur le budget et le règlement intérieur sont, après de nombreuses ratures successives, résumés en une formule lapidaire : “Adopté sans observation ${ }^{27}$ »... C'est sans doute ce que $\mathrm{R}$. Bouvier appelle « un heureux mélange de liberté et de dirigisme ${ }^{28} » .$. Mais sur qui s'exerçait cet «heureux mélange » et qui furent ses collaborateurs, ceux qui firent la Revue de synthèse historique?

«Cette revue est ouverte à tous ceux que son objet intéresse ${ }^{29}$ ", annonçait Henri Berr en 1900. Nombreux furent les intéressés puisque 196 personnes signèrent des publications de la Revue de synthèse historique durant sa première décennie. Toutefois, la grande majorité d'entre eux n'y collabora que très rarement : 113 une ou deux fois seulement et seuls 21 signèrent plus de dix parutions. Henri Berr à lui seul rédigea près du dixième des écrits...

Dans ces conditions, il serait illusoire d'établir un seuil de participation en deçà duquel les signataires occasionnels ne seraient pas considérés comme, collaborateurs (et ce d'autant plus que la diversité des rubriques comme des modes de sélection des publications rend aléatoire une classification fondée sur une hiérarchisation de l'implication de chacun en fonction du type

\footnotetext{
26. On évoquera, brièvement, les cas de Paul Hensel et Valory Le Ricolais : l'article du premier est publié avec un avertissement d'Henri Berr qui laisse entendré qu'il aurait pu ne pas être «publi[é] intégralement " pour cause de « réserves sur quelques points qui ne concordent pas avec les tendances de la Revue » $(R S H, t$, 7, oct. 1903, p. 163; combien d'auteurs furent dans ce cas?). Quant aux Bulletins critiques du second, ils expriment régulièrement sa difficulté à s'adapter au «cadre » (carcan?) de la revue et ce, jusqu'à l'interruption brutale de sa collaboration pourtant des plus régulières (pour une étude précise se reporter au mémoire cité supra n. 6).

27. Archives du Centre international de synthèse.

28. R. BOUVIER, art. cit. supra n. 23.

29. H. BERR, «Sur notre programme », art. cit. supra n. 7, p. 8.
} 
de texte signé). Aussi tous les signataires sont-ils pris en compte dans cette étude. Sur les 196, seuls 10 n'ont pu être un tant soit peu identifiés; les renseignements réunis sont aussi variés qu'inégaux mais suffisent à préciser la situation de la plupart d'entre eux et permettent, ainsi, d'englober dans une étude quantitative environ $90 \%$ des publications de la revue.

La Revue de synthèse historique entendait être une revue différente, réagissant contre une conception traditionnelle de l'histoire; c'est sans doute pourquoi elle réunit essentiellement de jeunes chercheurs : $70 \%$ de ses collaborateurs avaient entre 26 et 45 ans en 1900, les autres se répartissant presque équitablement entre plus jeunes et plus âgés (voir graphique : Âge des collaborateurs). La comparaison de 156 dates de naissance met en avant certaines années : 26 collaborateurs sont nés en 1861, 1862 ou 1863; 10 la seule année 1874. La tentation est grande d'y associer des promotions scolaires et universitaires: Abel Rey, l'un de ceux qui participa le plus à la revue, naquit en 1874 et, surtout, Henri Berr né en 1863, entra avec une dispense d'âge à l'École normale supérieure où il fut en contact avec des étudiants à peine plus vieux que lui ; 37 au moins des collaborateurs de la revue (soit plus d'un sur cinq) y furent élèves, et Henri Berr assurait que «beaucoup de [ses] camarades et de [ses] maîtres ${ }^{30}$ » avaient adhéré dès le départ à son projet. Nombre des rédacteurs de ce périodique furent élèves non seulement de l'École normale supérieure mais encore de l'École des chartes ou de l'École française de Rome ou d'Athènes et certains y étaient encore lorsqu'ils signèrent leur première publication. La jeunesse des collaborateurs resta une constante de la première décennie de la revue (voir graphique Âge des collaborateurs à leur arrivée à la revue). Si 40 d'entre eux avaient, lorsqu'ils y publièrent leur premier texte, l'âge d'Henri Berr lors de la fondation du périodique (entre 35 et 39 ans), les benjamins n'avaient pas plus de 20 ou 21 ans (L. Febvre était âgé de 27 ans lorsque Henri Berr lui demanda de rédiger une Région de la France). Durant ces dix années, 21 auteurs signèrent plus de 10 écrits, à deux exceptions près ${ }^{31}$, tous étaient nés entre 1861 et $1882 \ldots$

Ces collaborateurs apportaient à la revue ce qu'Henri Berr appela « leur autorité reconnue ou leur jeune ardeur ${ }^{32}$ ». H. Lévy-Bruhl vante « son génie (le mot n'est pas trop fort) de distinguer, pour collaborer à ses vastes entreprises, les meilleurs esprits, sans tenir compte de leurs titres académiques ou de leur situation sociale ${ }^{33} »$. Cependant, si nombre d'entre eux étaient

30. H. BERR, lettre à Gaston Milhaud, Revue de synthèse, t. 85, 1964, p. 103.

31. P. Lacombe et A.D. Xénopol.

32. H. BERR, «Au bout de dix ans », art. cit. supra n. 25, p. 5 et 6.

33. Henri LÉVY-BRUHL, Revue de synthèse, t. 85, 1964, p. 97-98. 
encore méconnus, les titres académiques ne leur faisaient pas défaut: 63 avaient déjà un doctorat lorsqu'ils signèrent à la revue et 40 l'eurent par la suite. Plus de la moitié des collaborateurs de la revue furent donc docteurs en lettres ou en droit, voire les deux. Ch.-V. Langlois sélectionne 34 thèses d'histoire qu'il considère comme les plus importantes soutenues entre 1892 et 1914 : 14 l'ont été par des collaborateurs de la Revue de synthèse historique ${ }^{34}$. D'ailleurs, parmi les 63 déjà titulaires d'un doctorat, 24 l'ont terminé entre 1900 et leur entrée à la revue : Henri Berr suivait de près les travaux universitaires. Comment aurait-il pu, sinon, maintenir le caractère novateur de son bimestriel?

«La Revue de synthèse historique a trouvé dans les universités beaucoup de ses meilleurs collaborateurs ${ }^{35} »$, déclara Henri Berr en 1910. Près de la moitié de ceux qui écrivirent dans la revue enseignèrent à l'université (88 exactement) sans compter ceux qui professèrent dans d'autres établissements supérieurs (28, du Collège de France à l'École des hautes études en passant par différentes écoles supérieures) ou dans les lycées (30). Soit près de 150 « universitaires » de la France entière : que les réunions de la rue Sainte-Anne se soient tenues le jeudi n'était pas un hasard. À ces enseignants s'ajoutaient essentiellement des conservateurs de musées, des archivistes, des bibliothécaires, des juristes, mais aussi quelques hauts fonctionnaires, un ingénieur ( $P$. Tannery) et un médecin ( $S$. Jankélévitch). Les différentes universités de province étaient représentées ainsi que les principaux lycées parisiens. Toutefois, on trouve ici aussi certaines dominantes : c'est ainsi que 10 professeurs du lycée Henri-IV écrivirent dans la revue. La présence de ces nombreux collègues d'Henri Berr met une fois de plus en valeur le rôle de ses cercles de relations et l'existence de discussions, de rencontres, de tout un fonctionnement imperceptible à la lecture du périodique.

Au-delà du monde universitaire français, la Revue de synthèse historique se tournait vers les autres pays et Henri Berr se félicitait d'y avoir attiré «un grand nombre de savants étrangers ${ }^{36} »$, expédiant leurs articles essentiellement d'Allemagne et d'Italie mais aussi de Suisse, d'Autriche, de Russie, des États-Unis, de Pologne, de Roumanie ou d'Angleterre ${ }^{37}$.

34. Charles-Victor LanGLoIs, «Les Études historiques», in La Science française, Paris, Larousse, 1915, p. 73-96.

35. Art. cit. supra n. 25 , p. 6.

36. H. BERR, lettre citée supra n. 9.

37.22 signataires. 
Les divers renseignements réunis sur les collaborateurs de la Revue de synthèse historique ne permettent pas toujours de déterminer avec certitude leur profession à la période qui nous intéresse et la relative polyvalence de ces universitaires - si restreinte soit-elle - accentue encore la difficulté. Il a cependant été possible dans 169 cas de déterminer leur formation ou du moins ce que nous appellerons leur activité essentielle. Ces collaborateurs sont les auteurs de $88 \%$ des textes publiés dans les vingt premiers tomes de la revue (soit 1105 sur 1261). Leurs activités essentielles ont été classées en sept catégories : Histoire et géographie; Archives, bibliothèques et musées; Langues et littératures françaises et étrangères; Philosophie; Sociologie; Psychologie; Droit. Une répartition qui permet d'étudier les parts respectives des différents types de chercheurs dans la production du périodique de synthèse historique.

\section{Répartition des collaborateurs et des publications par activité essentielle}

\begin{tabular}{|l|c|c|c|c|}
\hline \multirow{2}{*}{ Activité essentielle } & \multicolumn{2}{|c|}{ les collaborateurs } & \multicolumn{2}{c|}{ les parutions } \\
\cline { 2 - 5 } & nombre & $\%$ & nombre & $\%$ \\
\hline Histoire et géographie & 72 & 43 & 443 & 40 \\
\hline $\begin{array}{l}\text { Archives, bibliothèques } \\
\text { et musées }\end{array}$ & 12 & 7 & 133 & 12 \\
\hline $\begin{array}{l}\text { Langues et littératures } \\
\text { françaises et étrangères }\end{array}$ & 29 & 17 & 149 & 13,5 \\
\hline $\begin{array}{l}\text { Philosophie } \\
\text { dont Henri Berr }\end{array}$ & 32 & 19 & 331 & 30 \\
\hline Sociologie & 9 & 5 & 102 & 1,5 \\
\hline Psychologie & 5 & 3 & 16 & 1,5 \\
\hline Droit & 10 & 6 & 16 & 1,5 \\
\hline TOTAL & 169 & 100 & 1105 & 100,0 \\
\hline
\end{tabular}

Ce qui surprend d'abord, dans ces chiffres, c'est la faible quantité de sociologues et leur participation minime à la revue (aurait-on trop vite tiré des conclusions du célèbre débat Bouglé-Simiand ?), ainsi que, parallèlement, une relative importance du nombre de juristes (mais pour une participation tout aussi réduite). Ces chiffres, qui ne sont pas en rapport avec la production consacrée, par exemple, à la sociologie, montrent que certains chercheurs furent amenés à se pencher sur des domaines qui n'étaient pas les leurs.

Ensuite, on ne peut que reconnaître qu'on est loin des jugements émis 
par G. Monod et M. Siegel cités au début de cette étude. En effet, l'analyse quantitative montre exactement l'inverse : seuls $19 \%$ des collaborateurs de la Revue de synthèse historique furent des philosophes, contre $43 \%$ d'historiens, sans compter les archivistes (dont P. Lacombe), les littéraires, etc. Moins nombreux, les philosophes ont été des collaborateurs plus assidus que les historiens. Toutefois, si l'on excepte les écrits d'Henri Berr, classé ici parmi les philosophes ${ }^{38}$, le rapport n'est plus que de $22 \%$ des publications signées par des philosophes contre $44 \%$ par des historiens. Si on y ajoute les archivistes, bibliothécaires et conservateurs de musées, ce sont près des deux tiers des publications signées qui peuvent être imputées à des techniciens de la mémoire ${ }^{39}$ !

Organisée pour la synthèse, sous la férule d'un directeur mêlant « liberté et dirigisme », la Revue de synthèse historique intéressa de nombreux jeunes chercheurs qui y effectuèrent, surtout, un travail historique. Avec quels résultats et, au-delà des deux points de vue déjà cités, quel fut le regard extérieur porté sur cette revue?

Sa sortie fut peu saluée par les autres périodiques. Même la Revue historique, qui fut pourtant l'une de celles qui suivit au plus près ses livraisons successives, ne débuta son analyse qu'avec le deuxième numéro. Ce fut essentiellement à partir des années 1904-1905 que les publications de la Revue de synthèse historique commençèrent à être analysées par les autres périodiques ${ }^{40}$. Et encore. Cette revue n'est généralement prise en compte que d'une façon ponctuelle, lorsque l'une ou l'autre de ses parutions entre dans le cadre spécifique du périodique: histoire générale ou spécialisée, sociologie, philosophie, etc. Cela dit, que la Revue de synthèse historique ait été analysée partiellement et différemment selon les revues ne peut que conforter son image de publication différente...

Les manuels de bibliographie suivirent le même mouvement: l'édition de 1903 du Lehrbuch der historischen Methode und der Geschichtsphilosophie de E. Bernheim ne mentionne jamais Henri Berr ou la Revue de synthèse historique, celle de 1908 fait huit références à lui ou à sa revue - «die einzige Zeitschrift welche bisher das Gebiet der Methodologie spe-

38. Il signa près du tiers de leurs publications (102 sur 331) soit plus de $10 \%$ des écrits signés dans la revue.

39.576 textes sur 1003 soit $57,5 \% \ldots$

40. Un échantillon d'une dizaine de revues a été dépouillé : la Revue historique, la Revue d'histoire moderne et contemporaine, les Annales révolutionnaires, la Revue des questions historiques, L'Année sociologique, la Revue du mois, la Revue philosophique, la Revue critique, Etudes, la Nouvelle Revue et la Revue bleue. 
ziell vertritt ${ }^{41} \gg-c^{\prime}$ 'est-à-dire presque autant qu'à Ch.-V. Langlois (neuf fois) et plus qu'à É. Durkheim (six fois).

Dans l'édition de 1901 de son Manuel de bibliographie historique, Ch.-V. Langlois s'était montré sceptique face aux ambitions de la revue et notamment à propos des Revues générales "nécessairement analogues, disait-il, aux "bulletins", aux "courriers", aux "Berichte", et aux "Refatate" des autres revues d'histoire générale ». Il concluait sa présentation par un : «Nous verrons bien ${ }^{42}$.» En 1904, au contraire, il insiste sur la nécessité d'un bilan par spécialité de la production historique française et signale que c'est chose faite pour «plusieurs parties de l'histoire de France » dans... les Revues générales ${ }^{43}$. Il précise d'ailleurs que la Revue d'histoire moderne et contemporaine a « entrepris de son côté, la publication de "revues générales" qui ont le même caractère que celles de la Revue de synthèse historique $^{44}{ }^{\text {}}$. C'était simultanément reconnaître la spécificité des Revues générales et confirmer leur succès.

La revue d'Henri Berr s'était donc suffisamment imposée au bout de quelques années d'existence pour figurer sur les listes de périodiques importants, cités en exemple par les différents spécialistes. Suffisamment imposée pour qu'auteurs et éditeurs y espèrent une critique positive : le nombre de Bulletins critiques (rubriques consacrées aux «arrivages fortuits») va croissant et passe d'un minimum de huit dans le tome V (1902) à 66 dans le tome $\mathrm{XX}(1910)^{45} \ldots$

Ceux qui choisirent de collaborer à ce périodique avaient des conceptions théoriques extrêmement diverses. Toutefois, leur étude systématique ${ }^{46}$ permet de dessiner deux tendances qu'il serait erroné d'envisager comme deux écoles antagoniques. Tous revendiquent l'héritage des mêmes historiens et si les prises de position sont individuelles et particulières, toutes tournent autour d'une même problématique générale, toutes se résument à

41. Ernst BERNHEIM, Lehrbuch der historischen Methode und der Geschichtsphilosophie, Leipzig, Duncker und Humblot, 1908, p. 250.

42. Ch.-V. LangloIs, Manuel de bibliographie historique, Paris, Hachette, 1901, p. 178 et 179 .

43. Ch.-V. Langlois, op. cit., 1904, p. 405 et 406.

44. Ch.-V. LangloIs, op. cit. Cette rubrique fut publiée irrégulièrement sous le titre de Bibliographie critique. Philippe SAGNAC, signataire de la première, précise : «Il convient d'indiquer non seulement les livres qu'on possède, mais aussi et surtout les sources; [...] ce travail [...] pourra être utile aux historiens et leur servir de fil conducteur » (Revue d'histoire moderne et contemporaine, t. 4, 1902, p. 5). vantes.

45. Ils passent d'une moyenne de 12 par tome les cinq premières années à 42 les cinq sui-

46. Voir le mémoire cité supra n. 6. 
une simple question : « Analyse ou synthèse ? » L'historien devait-il se cantonner dans l'analyse ou tendre à la synthèse ? Quand fallait-il débuter celle-ci? Quelle discipline devait s'y attacher? Et quelles études historiques entreprendre pour quelle fonction de l'histoire?

Les réponses apportées ne peuvent être considérées comme contradictoires, ce sont les limites, les bornes de chaque étape, de chaque discipline qui sont en jeu. Les uns cantonnaient l'histoire dans un tableau chronologique, les autres l'élargissaient à l'étude des similitudes entre les faits, indépendamment de leur localisation temporelle ou géographique. D'aucuns entendaient épuiser l'analyse avant de synthétiser; d'autres défendaient le besoin de synthèses provisoires pour guider l'analyse et éviter pertes de temps et efforts inutiles. Certains se satisfaisaient de la recherche et de la présentation des faits éprouvés tandis qu'ils laissaient le soin de les généraliser à d'autres disciplines - la sociologie notamment - alors que les autres revendiquaient le droit de mener l'étude amorcée à son terme : la synthèse. Enfin, ils considéraient l'histoire soit comme un instrument de culture intellectuelle, soit comme la discipline qui devait guider l'humanité.

Mais ces opinions n'étaient pas contradictoires, elles allaient dans le même sens. Si l'on excepte les rares tenants d'une histoire artistique, tous considéraient les faits issus de l'analyse érudite comme des faits scientifiques. Tous se référaient finalement aux mêmes définitions de l'histoire (la science des faits humains du passé) et de la science (il n'y a de science que du général) et visaient la généralisation de leurs résultats. Tous adoptaient la même démarche méthodique. Tous mettaient l'histoire en bonne place dans la hiérarchie des sciences et refusaient d'envisager son inutilité. Seules variaient les limites.

«Analyse ou synthèse? ? : fallait-il épuiser l'analyse ou amorcer des synthèses provisoires? (Quand fallait-il amorcer la synthèse ?) L'historien devait-il généraliser ou était-ce la tâche du sociologue ? (Qui devait généraliser?) L'historien devait-il attendre que les conclusions de ses recherches influent sur l'avenir ou devait-il s'engager sur le terrain politique? (Jus$q u$ 'où allait la tâche de l'historien?).

La Revue de synthèse historique était donc moins une réaction contre la pratique historique contemporaine qu'une tentative de dépassement. Elle n'entendait pas inverser le courant, prendre le contre-pied de ce qui se pratiquait couramment, elle voulait aller plus loin, plus vite et plus précisément. Elle voulait aller au-delà. Elle prétendait déplacer les limites.

Aussi les historiens qui, au début du siècle, critiquèrent l'histoire traditionnelle, celle qui était enseignée et montrée en exemple, se réunirent-ils autour d'Henri Berr et de sa revue. L. Febvre présenta son choix de s'ins- 
crire «parmi les fidèles de la Revue de synthèse historique » comme une aventure: ce bimestriel était le «cheval de Troie par quoi s'insinuèrent dans la place tant de nouveautés ennemies et troublantes ${ }^{47}$ ». Mais il écrivit également à propos de ceux qui participèrent activement à la Revue de synthèse historique: "Ceux-là préparaient l'avenir sans doute; ils ne mâ̂trisaient certes pas le présent ${ }^{48}$. . Cette revue fut, toutefois, beaucoup plus qu'une «sorte de préhistoire théorique des Annales ${ }^{49}$ ». Le rôle novateur de ces dernières (souvent présentées comme une réaction soudaine contre l'histoire de Lavisse, Monod, Langlois et Seignobos) serait dû essentiellement aux points suivants : remise en question du cloisonnement disciplinaire en faveur de l'interdisciplinarité ; prise de position contre la chronologie fine, en faveur de la longue durée et de perspectives diachroniques; option pour un élargissement de l'histoire vers la géographie humaine, l'économie et la sociologie contre une histoire limitée aux événements; relativisation du fait historique. Seul ce dernier point n'était pas dans le programme de la Revue de synthèse historique (toutefois l'importance attribuée à la synthèse provisoire comme état de la recherche, hypothèse susceptible d'être modifiée, et donc nécessairement conviction provisoire, ne peut-elle être envisagée comme un signe précurseur de cette relativité ?).

La Revue de synthèse historique fut pourtant confrontée à un échec, un échec d'autant plus cuisant qu'il concerne l'un des points clés de son programme, un de ses objectifs les plus importants, une des idées majeures d'Henri Berr : la collaboration. En effet, les propositions d'Enquêtes avortèrent ou n'aboutirent pas, faute de réponses.

Régions de la France ou Revues générales devaient être les «synthèses partielles » de "synthèses plénières », or celles-ci ne furent jamais réalisées; elles restent des études partielles n'ayant en commun qu'une méthodologie pour les unes, une problématique pour les autres. Quant à la réelle coopération entre chercheurs, celle qui les amène à travailler ensemble sur un même sujet, seules deux études - parmi les 201 Articles de fond, 50 Revues générales et sept Régions de la France publiés dans les vingt premiers tomes de la revue - furent réalisées collégialement par deux chercheurs ${ }^{50}$... Si la Revue de synthèse historique contribua à multiplier les contacts entre spécialistes, la recherche historique n'en resta pas moins une pratique individuelle, faute d'être une pratique solitaire. La seule forme

47. Lucien Febvre, Combats pour l'histoire, Paris, A. Colin, 1953, p. vII et 393.

48. Ibid., p. 10. Ce texte est également publié dans la Revue de synthèse, t. 57, 1934, p. 100.

49. André BuRGuière, «La notion de "mentalités" chez M. Bloch et L. Febvre», Revue de synthèse, t. 104,1983 , p. 333 et 334.

50. Il s'agit de $\mathrm{N}$. Vaschide et $\mathrm{H}$. Pieron (deux psychologues) et de J. Calmette et P. Vidal (respectivement archiviste-paléographe et conservateur). 
de coopération courante restait une «collaboration passive »: utilisation par un travailleur isolé des résultats d'autres travailleurs isolés...

Si G. Monod considérait Henri Berr comme un philosophe se mêlant d'histoire, la rubrique nécrologique publiée cinquante ans plus tard dans sa Revue historique (après la Revue de synthèse, «L'Évolution de l'humanité », le Centre international de synthèse, etc.) juge indispensable d'informer le lecteur qu'Henri Berr qui avait «consacré à la rénovation des études historiques la majeure partie de sa vie, n'avait pas, comme on le croit souvent, professé l'histoire ${ }^{51}$ " : les héritiers de Gabriel Monod considéraient Henri Berr comme un historien...

Pourquoi, alors, considérer aujourd'hui la Revue de synthèse historique comme une revue de philosophes à laquelle collaborèrent épisodiquement quelques historiens? Parce que des philosophes signèrent la plupart des articles théoriques. Parce que ces articles, qui ont moins vieilli que les études concrètes, sont plus susceptibles d'intéresser le lecteur contemporain. Parce que l'abondance des écrits d'Henri Berr peut accentuer encore cette impression de prépondérance de la philosophie. Mais ce n'est qu'une impression. L'étude quantitative en atteste : de nombreux historiens, archivistes, bibliothécaires, linguistes, littéraires firent dans la revue un abondant travail historique pratique. La préoccupation théorique ou méthodologique est présente dans la plupart des Articles de fond, Revues générales, Régions de la France, études bibliographiques, etc., mais elle y figure en plus de l'étude concrète de l'histoire. C'était une revue de synthèse, mais de synthèse historique, qui entendait s'opposer à la philosophie de l'histoire, qui revendiquait sa scientificité.

La Revue de synthèse historique était donc en 1910 une revue historique reconnue pour ses travaux historiques, pour ses études synthétiques de l'histoire, pour sa pratique historique. Elle était «l'organe spécial » de théorie et de méthodologie historique; sa prééminence dans ce domaine n'était pas mise en cause, mais ce n'est pas cette part de sa production qui assura son succès, ce n'est pas elle que ses contemporains mirent en valeur. C'est elle que retint l'historiographie.

Si tous sont unanimes à reconnaître l'importance de la discussion au sein du périodique de «Synthèse historique »; si de nombreux penseurs eurent l'occasion d'y discuter et d'y être discutés, un seul regretta de ne pas l'avoir été suffisamment: Henri Berr. Il écrivit, juste avant sa mort: «Beaucoup d'idées ont cheminé qui inspiraient en 1900 la Revue de synthèse historique

51. Louis-Philippe LE MAY, in Revue historique, t. 213, 1955, p. 201. 
[...] mais notre conception de la Synthèse scientifique ${ }^{52} n$ 'a été $[\ldots]$ ni pleinement appliquée, ni même sérieusement discutée. Il semble que de cette "Synthèse en histoire" on ait apprécié le détail et négligé l'essentiel ${ }^{53}$."

La Revue de synthèse historique était organisée par Henri Berr autour de sa personne : il sut y intéresser de nombreux chercheurs, dans des domaines extrêmement variés; il sut aménager son périodique pour que théorie et pratique puissent s'y côtoyer, s'y combiner. Mais il ne sut pas imposer ses options philosophiques. Pourquoi ? À cause du trop grand nombre de collaborateurs, de l'éventail des domaines envisagés, etc. Dans l'ouverture de son périodique aux différentes théories, la sienne ne parut plus qu'une conception parmi d'autres. Une pensée trop générale, trop synthétique (systématique?) peut-être, pour intéresser nombre d'historiens, mais surtout trop sous-jacente. Henri Berr s'investit dans sa revue surtout comme directeur, comme organisateur, non comme théoricien. Il était convaincu que la discussion des différents points de vue suffirait à imposer le $\operatorname{sien}^{54}$. Elle ne l'imposa pas.

Pour être reconnu par les historiens, Henri Berr distingua nettement, dans ses écrits et ses activités, le philosophe de l'historien. Cette distinction perdura: les historiens ne s'intéressèrent pas aux convictions du premier mais adoptèrent petit à petit les idées, les méthodes du second. Il fut relayé par de jeunes historiens convaincus du bien-fondé de ses opinions sur l'histoire, peu préoccupés par sa théorie philosophique. Ceux-ci promurent et développèrent ses convictions quant à la pratique de la discipline historique mais ils négligèrent ses convictions philosophiques, ils ne se soucièrent pas de la «Synthèse historique ${ }^{55}$. Et la Revue de Synthèse historique ne promut que la synthèse en histoire, une nouvelle façon de concevoir l'histoire. Une Nouvelle Histoire.

52. Henri Berr a renoncé alors au terme de Synthèse historique : changement d'étiquette dans l'espoir d'une meilleure compréhension mais non évolution de sa conception.

53. H. BERR, "Appendice " de la rééd. de La Synthèse en histoire, Paris, A. Michel, 1953, p. 307-308. C'est moi qui souligne. Henri Berr poussa cette envie d'être discuté jusqu'à l'absurde. Ainsi, il commenta et cita abondamment et dans la rubrique Notes, questions et discussions une communication faite à une séance de la Société d'histoire moderne parce qu'elle " résum[ait] et sur un ou deux points complét[ait] des articles et notes publiés dans la revue »: il analysait d'après le bulletin de cette société (sic) une communication faite par... « M. Henri Berr $"$ ! (RSH, t. 10,1905 p. $369-372)$

54. Toute la conception philosophique d'Henri Berr reposant sur l'idée que la vérité s'impose d'elle-même, progressivement, comment la discussion aurait-elle pu mener à d'autres conclusions que les siennes puisqu'il était dans le vrai?

55. La «Synthèse historique » est d'ailleurs rarement appréhendée comme une discipline en soi : le peu de respect pour la typographie adoptée par Henri Berr (majuscule-minuscule) en atteste. Quant à sa création la plus connue du grand public, «L'Évolution de l'humanité », les dernières rééditions ne comportent même plus ses préfaces... 